\title{
Homogeneous nucleation in associated vapors. III. Heptanoic, decanoic, and myristic acids
}

\author{
Gopal Agarwal
}

Richard H. Heist

Fairfield University, rheist@fairfield.edu

Follow this and additional works at: https://digitalcommons.fairfield.edu/engineering-facultypubs

(C) 1980 American Institute of Physics.

The final publisher PDF has been archived here with permission from the copyright holder.

\section{Peer Reviewed}

\section{Repository Citation}

Agarwal, Gopal and Heist, Richard H., "Homogeneous nucleation in associated vapors. III. Heptanoic, decanoic, and myristic acids" (1980). Engineering Faculty Publications. 152.

https://digitalcommons.fairfield.edu/engineering-facultypubs/152

\section{Published Citation}

Agarwal, G., \& Heist, R. H. (1980). Homogeneous nucleation in associated vapors. III. Heptanoic, decanoic, and myristic acids. The Journal of Chemical Physics, 73(2), 902-907. doi:10.1063/1.440198.

This item has been accepted for inclusion in DigitalCommons@Fairfield by an authorized administrator of DigitalCommons@Fairfield. It is brought to you by DigitalCommons@Fairfield with permission from the rightsholder(s) and is protected by copyright and/or related rights. You are free to use this item in any way that is permitted by the copyright and related rights legislation that applies to your use. For other uses, you need to obtain permission from the rights-holder(s) directly, unless additional rights are indicated by a Creative Commons license in the record and/or on the work itself. For more information, please contact digitalcommons@fairfield.edu. 


\title{
Homogeneous nucleation in associated vapors. III. Heptanoic, decanoic, and myristic acids
}

\author{
Gopal Agarwal and Richard H. Heist ${ }^{a)}$ \\ Department of Chemical Engineering, University of Rochester, Rochester, New York 14627 \\ (Received 7 February 1980; accepted 4 April 1980)
}

Homogeneous nucleation measurements have been made on heptanoic, decanoic, and myristic acid vapors, all of which exhibit small degrees of hydrogen bond association. The measured temperature dependences of the critical supersaturation are compared with predictions of the Katz-Saltsburg-Reiss theory for nucleation in associated vapors as well as the conventional Becker-Döring-Zeldovitch theory. Heptanoic acid and decanoic acid show good agreement with theory while the myristic acid critical supersaturation measurements are consistently low.

\section{INTRODUCTION}

There has been considerable interest recently in particulate formation in the presence of reacting vapors. Areas of interest include $\mathrm{H}_{2} \mathrm{O}-\mathrm{H}_{2} \mathrm{SO}_{4}$ binary nucleation as a source of atmospheric aerosol, particulate formation in vapors during coal gasification, and particulate formation in steam turbine and gas turbine systems. While heterogeneous processes most certainly play an important role in all of the areas, there is good reason to believe homogeneous nucleation may be important as well. One example is the profound effect $\mathrm{H}_{2} \mathrm{SO}_{4}$ has on the vapor phase homogeneous nucleation of $\mathrm{H}_{2} \mathrm{O}$. Model calculations, ${ }^{1-4}$ which include the $\mathrm{H}_{2} \mathrm{SO}_{4}$ hydrate equilibrium reaction, predict homogeneous binary $\mathrm{H}_{2} \mathrm{O}-\mathrm{H}_{2} \mathrm{SO}_{4}$ nucleation at finite rates even in vapors undersaturated with respect to the pure components, e.g., $50 \% \mathrm{RH}$ for water $a_{2}=10^{-3}$ for $\mathrm{H}_{2} \mathrm{SO}_{4}$. (Note, unit activity corresponds to an $\mathrm{H}_{2} \mathrm{SO}_{4}$ partial pressure of $3.6 \times 10^{-4} \mathrm{Torr}$, see Ref. 3 for details. ) In addition, these calculations reveal that the presence of the hydrate equilibrium has a stabilizing effect upon the vapor with respect to condensation, that is to say, the rate of homogeneous nucleation decreases in a vapor which supports the hydrate equilibrium. These calculations are in good agreement with existing experimental data. ${ }^{4,5}$

In order to better understand the role of chemical reaction in nucleation, we are currently investigating the conditions necessary for homogeneous nucleation in reacting vapor systems. The first model system chosen for our study was the family of carboxylic acids. While somewhat inconvenient to work with, these acids do have a number of useful properties. First of all, the chemical reaction is the vapor phase monomer-dimer hydrogen bond association reaction and is reasonably well understood. Secondly, the number of components in our experiment we have to concern ourselves with is small, three at most. Third, the degree of reaction is variable; the reaction can be turned on or off by choosing the appropriate carboxylic acid. Finally, the results of our measurements can be compared with various (conflicting) theories of nucleation in associated vapors presently in the literature.

In the first two papers of this series $^{6,7}$ we reported

a) To whom all correspondence should be addressed. measurements of the variation of the critical supersaturation with temperature for formic, acetic, and propanoic acids. All three of these acids are heavily associated in the vapor phase (for example, dimer mole fractions at critical supersaturation and $290 \mathrm{~K}$ are 0.91 , 0.93 , and 0.77 , respectively), and the results of these investigations show clearly that the presence of the association equilibrium stabilizes the vapor with respect to condensation. Equivalently, the rate of homogeneous nucleation is decreased in a vapor which supports the association equilibrium. In addition, we observed that the predictions of the Katz-Saltsburg-Reiss theory for homogeneous nucleation in associated vapors ${ }^{8}$ follow most closely our measured values. The expression for the homogeneous nucleation rate in the Katz-SaltsburgReiss theory is

$$
\begin{aligned}
J= & \frac{a}{d}\left(\frac{2 N_{0}^{3} \sigma M}{\pi}\right)^{1 / 2}\left(\frac{P_{e} S}{R T}\right)^{2} \\
& \times \sum_{i=1}^{m} i^{3 / 2} x_{i} \exp \left[-\frac{16 \pi N_{0}}{3\left(\ln S_{1}\right)^{2}}\left(\frac{M}{d}\right)^{2}\left(\frac{\sigma}{R T}\right)^{3}\right] .
\end{aligned}
$$

In this equation, $J$ is the nucleation rate in drops/ $\mathrm{cm}^{3} \mathrm{sec}, a$ is the condensation coefficient (taken as unity in our calculations), $d$ is the liquid density, $N_{0}$ is Avogadro's number, $\sigma$ is the bulk liquid surface tension, $M$ is the molecular weight, $P_{e}$ is the equilibrium vapor pressure, $S$ is the supersaturation ratio (defined as $P / P_{e}$ with $P$ being the existing pressure of the vapor), $R$ is the gas content, $i$ is the number of molecules in an association cluster of size $i, x_{i}$ is the ratio $P_{i} / P$ (with $P_{i}$ being the partial pressure of the $i$-size clusters and $P$ the actual pressure of the vapor), and $S_{1}$ is the monomer supersaturation ratio $P_{1} / P_{1 e}$ (where $P_{1 e}$ is the equilibrium vapor pressure of the monomer only).

In these experiments with formic, acetic, and propanoic acids the degree of stabilization is significant. For instance, in Fig. 3 of Ref. 6 at $300 \mathrm{~K}$ the experimental critical supersaturation is nearly twice the critical supersaturation predicted by the Becker-DöringZeldovitch theory for the pure monomer vapor. This represents a decrease in the monomer nucleation rate of about $10^{18}$.

Since the presence of the association reaction is observed to decrease the homogeneous nucleation rate (increase the critical supersaturation), the next step is to 
reduce the association reaction and repeat the critical supersaturation measurements. This is done by using other members of the carboxylic acids exhibiting smaller degrees of association. In what follows we present experimental data on the temperature variation of the critical supersaturation of heptanoic, decanoic, and myristic acids $\left(C_{7}, C_{10}\right.$, and $C_{14}$, respectively). Each of these acids is either known to or expected to undergo a small degree of vapor phase association.

\section{EXPERIMENT DESCRIPTION}

The critical supersaturation measurements described in this paper were made using an upward thermal diffusion cloud chamber. Description of the thermal diffusion cloud chamber as well as the principles of operation have been given elsewhere and will not be reproduced in detail here. ${ }^{6-9}$ The diffusion cloud chamber used for the experiments described in this paper is, in fact, the same chamber described in Ref. 7 with one minor variation, the surfaces of both chamber plates are uncoated, highly polished aluminum whereas the surface of the lower plate of the cloud chamber described in Ref. 7 was Teflon coated. The temperatures of the upper plate and lower plate liquid surfaces were measured either directly, by bringing thermocouples into the cloud chamber through the Pyrex ring, or indirectly by first measuring the surface temperatures of the cloud chamber plates and then calculating the temperature difference across the liquid layers. Our results were the same in either case. A dc electric field was applied across the cloud chamber plates to sweep out ions resulting from cosmic ray or naturally occurring radioactive sources. During all of the critical supersaturation measurements either a 650 W tungsten-halide lamp or a $2 \mathrm{~mW}$ helium-neon laser were used to observe the nucleation. Helium gas was used as the carrier gas for all of our critical supersaturation measurements, and Viton gaskets were used to separate the Pyrex ring from both cloud chamber plates.

Briefly, during operation of the cloud chamber, the working fluid evaporates from the lower plate pool maintained at a higher temperature and diffuses through a light, inert carrier gas to the upper plate where it condenses at a lower temperature forming a film which drains slowly to the inside edge of the Pyrex ring and down the wall to the pool on the lower plate. The light carrier gas is necessary to maintain a decreasing density gradient from the lower to the upper plate thus preventing natural convection. When properly run, the cloud chamber operates in a quiescent, steady-state mode with very nearly plane-parallel diffusion of the working fluid vapor. To determine the vapor supersaturation and the temperature inside the diffusion cloud chamber, it is necessary to solve the coupled energy and mass transport equations which describe the mass and energy fluxes between the two chamber surfaces.

If we neglect all effects of thermal diffusion, all contributions to diffusion arising from external forces and gradients, assume a perfect gas mixture of acid monomer, acid dimer, and carrier gas, and assume local equilibrium throughout the chamber, it can be shown that (for the steady-state, plane-parallel diffusion con- ditions which prevail in the chamber) the pressure and temperature profiles can be written as

$$
\frac{d P}{d Z}=\frac{\left(P-P_{T}\right) \beta}{C D_{13}} L
$$

and

$$
\frac{d T}{d Z}=\lambda^{-1}(L H-Q)
$$

respectively. ${ }^{6}$ In Eq. (2), $P=P_{1}+P_{2}$ is the total partial pressure of the diffusing species (monomer plus dimer), $P_{T}$ is the total chamber pressure, $Z$ is the reduced chamber height, $z / h$, where $h$ is the actual chamber height, $C$ is the total concentration, and $D_{13}$ is the monomer-carrier gas binary diffusion coefficient. $L$ is the reduced molar flux given by $(l h) / M_{1}$, where $l=l_{1}+l_{2}$ is the total mass flux ( $z$ direction) and $M_{1}$ is the molecular weight of the monomer, and $\beta=1-0.29 \phi$. Here, $\phi=l_{2} /$ $l$ is the dimer flux fraction. In Eq. (2) $\lambda$ is the mixture thermal conductivity, and $Q$ is the reduced energy flux $q h$, with $q$ being the energy flux, and $H=H_{1}+\phi\left(H_{2} / 2+H_{1}\right)$, where $H_{1}$ and $H_{2}$ are the monomer and dimer molar enthalpies, respectively. The derivation and the solution of these equations which give the pressure and temperature profiles in the chamber are given elsewhere. ${ }^{6}$ The values of the various thermodynamic and hydrodynamic properties used in the solution of Eqs. (2) and (3) for heptanoic, decanoic, and myristic acids are summarized in Table I. An increasingly serious problem with diffusion cloud chamber experiments on these more complex chemical systems is the lack of property data necessary to evaluate the coefficients in the transport equations. ${ }^{6,7,10}$ For instance, equilibrium vapor pressure data below 1 Torr, monomer-dimer equilibrium association constants, and data on the vapor thermal conductivities for these higher molecular weight carboxylic acids are very difficult to find. We shall return to this problem later.

The decanoic and myristic acids used in this investigation were manufactured by the Aldrich Chemical Company. The heptanoic acid was manufactured by Eastman Kodak and was quoted as being $98 \%$ pure while the decanoic and myristic acids were quoted as being at least 99\% pure. AIRCO, 99. 995\% helium gas was used as the carrier gas for all of our experiments. No attempt was made to purify either the helium carrier gas or the carboxylic acids further. Thermocouple voltages were measured with a Leeds and Northrup $\mathrm{K}-2$ potentiometer, and trends in the thermocouple voltages were followed using the Houston Omniscribe chart recorder. Total pressure measurements were made using a Heise model CMM pressure gauge.

\section{RESULTS AND DISCUSSION}

The experimental data obtained for heptanoic, decanoic, and myristic acids is listed in Table II. In Table II, $T_{0}$, the surface temperature of the working fluid pool on the lower cloud chamber plate is listed, as well as the upper plate film surface temperature $T_{1}$, the total pressure $P_{T}$, and the pressure ratio $P_{r}$, for each experiment. 
TABLE I. Expressions for the heptanoic acid, decanoic acid, and myristic acid thermal conductivity $\lambda_{e}$, saturation vapor pressure $P_{e}$, surface tension $\sigma$, vapor viscosity $\eta$, density $d$, monomer molar heat capacity at constant pressure $c_{p 1}$, dimer molar heat capacity at constant pressure $c_{p 2}$. The expression for the heptanoic acid association equilibrium constant, $K_{2}$, and values for the monomer molecular weight $M$, binary diffusion coefficient $D_{13}$, and the binary diffusion coefficient temperature dependence $s$, for each acid. Expressions for the carrier gas thermal conductivity $\lambda_{e}$, and vapor viscosity, $\eta$, as well as the carrier gas moleculer weight. ${ }^{2}$

Neptanoic acid:

$$
\begin{aligned}
& \lambda_{e}=\left(0.0473+1.65 \times 10^{-3} T\right)\left[\frac{9.688 \times 10^{-6} T^{1.5}}{T+727}+0.375 T\right] \\
& +0.375 T^{-1.25}\left[\frac{x_{1} x_{2}}{\left(2 x_{2}+x_{1}\right)^{2}}\right]^{b} \\
& \log _{10} P_{e}=8.352-\frac{2312.4}{(T-70.838)} \\
& \sigma=29.708-0.085683(T-283.2)^{\mathrm{d}} \\
& \eta=\frac{9.688 \times 10^{-6} T^{1.5 e}}{(T+727.0)} \\
& d=1.16888-8.44937 \times 10^{-3} T^{\mathcal{P}} \\
& c_{p 1}=3.987+1.629 \times 10^{-1} T-9.394 \times 10^{-5} T^{3 \mathrm{~g}} \\
& c_{p 2}=7.973+3.258 \times 10^{-1} T-1.879 \times 10^{-4} T^{3} \mathrm{~g} \\
& \log _{10} K_{2}=\frac{2000}{T}-7.42^{\mathrm{h}} \\
& M=130.19 ; \mathrm{He} D_{13}=0.2026^{1} \\
& s=0.75^{1}
\end{aligned}
$$

Decanoic acid:

$$
\begin{aligned}
& \lambda_{e}=\left(0.0448+1.739 \times 10^{-3} T-1.007 \times 10^{-6} T^{2}\right) \frac{\left[9.0668 \times 10^{-6} T^{1.5}\right]^{j}}{(T+796.2)} \\
& \log _{10} P_{e}=7.9458-\frac{2203.2}{(T-106.4)} \\
& \sigma=30.46-0.0762(T-273.2)^{1} \\
& \eta=9.0668 \times 10^{-6} T^{1.5} /(T+796.2)^{e} \\
& d=0.91788-7.7131 \times 10^{-4}(T-273.2)^{1} \\
& c_{p 1}=5.1702+0.22697 T-1.2985 \times 10^{-4} T^{2}+2.8789 \times 10^{-8} T^{3} \\
& M=172.26 ; \mathrm{He} D_{13}=0.1686^{1} \\
& s=0.75^{1}
\end{aligned}
$$

\section{Myristic acid:}

$$
\begin{aligned}
& \lambda_{e}=-1.8283 \times 10^{-6}+1.1788 \times 10^{-8} T+2.2396 \times 10^{-10} T^{2} \\
& -1.2186 \times 10^{-13} T^{3 \mathrm{~m}} \\
& \log _{10} P_{e}=8.2271-\frac{2536.2}{(T-114.5)}^{\mathrm{n}} \\
& \sigma=33.061-7.9836 \times 10^{-2}(T-273.2) \\
& \eta=\frac{6.7293 \times 10^{-6} T^{1.5 \mathrm{~m}}}{(T+425.31)} \\
& d=0.90097-7.1406 \times 10^{-4}(T-273.2)^{\circ} \\
& c_{p 1}=6.7482+3.1242 \times 10^{-1} T-1.7773 \times 10^{-4} T^{2}+3.9173 \times 10^{-8} T^{3} \\
& M=228.38 ; \mathrm{He}_{\mathrm{e}} D_{13}=0.1379^{\mathrm{P}} \\
& s=0.75^{\text {D }}
\end{aligned}
$$

\section{TABLE I (Continued)}

Helium:

$$
\begin{aligned}
& \lambda_{c}=7.376974 \times 10^{-5}+1.39222 \times 10^{-6} T-6.343536 \times 10^{-10} T^{2} Q \\
& \eta=\frac{145.5 \times 10^{-7} T^{1.5} 1}{(T+74.1)}
\end{aligned}
$$

$M=4.0026$

${ }^{2} \lambda_{e}$ in $\mathrm{cal} /(\mathrm{cm} \mathrm{s} \mathrm{K}) ; P_{e}$ in $\mathrm{mm} ; \sigma$ in $\mathrm{erg} / \mathrm{cm}^{2} ; \eta$ in poise; $d$ in $\mathrm{g} / \mathrm{cc} ; c_{p}$ in cal/(mol K); $K_{2}$ in $\mathrm{mm}^{-1} ; D_{13}$ in $\mathrm{cm}^{2} / \mathrm{s}$ at $273.16 \mathrm{~K}$ and $1 \mathrm{~atm}, s$ is dimensionless; $T$ in $\mathrm{K}$.

'The first term is the conductivity of pure monomer $\lambda_{f}$, and the second term is the conductivity due to chemical reaction $\lambda_{R}$. $\lambda_{f}$ was determined using the modified Eucken correction; viscosity data was estimated using the method described in

Perry's Chemical Engineers' Handbook, 4th ed. (McGraw-Hill, New York, 1963), pp. 3-229; $\lambda_{R}$ was determined by the method described in R. H. Heist, K. M. Colling, and C. S. Dupuis, J. Chem. Phys. 65, 5147 (1976).

'The expression for the equilibrium vapor pressure was obtained from data given in E. Jantzen and W. Erdman, Fette und Seifen 54, 197 (1952) and in D. H. Stull, Ind. Eng. Chem. 39, 517 (1947).

The surface tension for heptonic acid was estimated from Caporic acid $\left(C_{6}\right)$ and Caprylic acid $\left(C_{8}\right)$ data given by K. W Hunten and O. Maass, J. Am. Chem. Soc. 51, 153 (1929). The expression for the vapor viscosity data was obtained using A rnold's estimation technique described in Perry's Chemical Engineers' Handbook, 4th ed. (McGraw-Hill, New York, 1963), pp. 3-229.

${ }^{\text {IT }}$ The density was estimated from data given by $\mathrm{K}$. W. Hunten and O. Maass, J. Am. Chem. Soc. 51, 153 (1929) and from data found in the International Critical Tables (1929).

Estimated using the group contribution method of D. N. Rihani and L. K. Doraiswamy, Ind. Eng. Chem. (Fund.) 4, 17 (1965). ${ }^{\mathrm{h}}$ T. M. Fenton and W. E. Garner, J. Chem. Soc. 694 (1930). ${ }^{1}$ Estimated using the method of E. N. Fuller, P. D. Schettler and J. C. Giddings, I \& EG 58, 19 (1966).

'See footnote $b$ for pure monomer case.

${ }^{k}$ The equation for the equilibrium vapor pressure was obtained from data given by E. Jantzen and W. Erdmann, Fette und Seifen 54, 197 (1952).

${ }^{1}$ Estimated from data given by $\mathrm{K}$. W. Hunten and $\mathrm{O}$. Maass, J. Am. Chem. Soc. 51, 153 (1929).

mThe expressions for $\lambda_{e}$ and $\eta$ were estimated using the method described in J. O. Hirschfelder, C. F. Curtiss, and R. B. Bird, Molecular Theory of Gases and Liquids (Wiley, New York, 1954), Chap. 8.

${ }^{\mathrm{n}}$ The expression for the equilibrium vapor pressure was obtained from data given by E. Jantzen and W. Erdmann, Fette und Seifen 54, 197 (1952) and E. Hammer and A. L. Lydersen, Chemical Engineering Science 7, 66 (1957).

JJ. M. Costello and S. T. Bowden, Rec. Trav. Chim. 77, 803 (1958).

${ }^{D}$ E. N. Fuller, K. Ensley, and J. C. Giddings, J. Phys. Chem 73, 3679 (1969).

'Thermophysical Properties Research Center Data Book (Purdue University, 1970), Vol. 3.

The pressure ratio, defined as the total pressure divided by the equilibrium vapor pressure at $T_{0}$, turns out to be a useful criterion for maintaining stability inside the cloud chamber. For example, at values of $P_{r}$ much below 2 or 3 for many substances the mass flux in the chamber increases significantly so as to render uncertain the actual value of the pressure and temperature profiles inside the chamber and, consequently, the 
TABLE II. Experimental data for the heptanoic acid, decanoic acid and myristic acid critical supersaturation measurements. $T_{0}$ is the surface temperature of the working fluid, $T_{1}$ is the surface temperature of the condensate film on the upper plate, $P_{T}$ is the total pressure inside the cloud chamber. $P_{r}$ is the pressure ratio, calculated as the ratio of the total pressure $p_{T}$ and the equilibrium pressure of the working fluid at $T_{0}$.

\begin{tabular}{|c|c|c|c|c|}
\hline $\begin{array}{l}\text { Experiment } \\
\text { No. }\end{array}$ & $T_{0}(\mathrm{~K})$ & $T_{1}(\mathrm{~K})$ & $P_{T}(\mathrm{mmHg})$ & $P_{r}$ \\
\hline \multicolumn{5}{|c|}{ Heptanoic acid } \\
\hline 1 & 359.0 & 301.8 & 27 & 12.7 \\
\hline 2 & 370.2 & 312.3 & 47 & 11.1 \\
\hline 3 & 380.3 & 321.5 & 58 & 7.63 \\
\hline 4 & 396.1 & 338.4 & 94 & 5.37 \\
\hline 5 & 407.8 & 350.8 & 263 & 8.50 \\
\hline 6 & 422.1 & 362.9 & 330 & 5.62 \\
\hline 7 & 431.8 & 377.0 & 518 & 5.89 \\
\hline 8 & 452.5 & 396.8 & 741 & 3.77 \\
\hline \multicolumn{5}{|c|}{ Decanoic acid } \\
\hline 1 & 390.3 & 325.7 & 12 & 7.82 \\
\hline 2 & 391.5 & 328.7 & 11 & 6.67 \\
\hline 3 & 397.0 & 334.0 & 14 & 6.06 \\
\hline 4 & 401.1 & 338.6 & 18 & 6.10 \\
\hline 5 & 409.1 & 343.6 & 18 & 3.87 \\
\hline 6 & 417.4 & 351.2 & 28 & 3.86 \\
\hline 7 & 422.9 & 357.4 & 48 & 4.98 \\
\hline 8 & 434.1 & 364.2 & 54 & 3.24 \\
\hline 9 & 440.5 & 374.4 & 102 & 4.53 \\
\hline 10 & 445.1 & 378.3 & 132 & 4.77 \\
\hline 11 & 450.9 & 385.3 & 208 & 5.85 \\
\hline 12 & 458.1 & 391.0 & 229 & 4.76 \\
\hline 13 & 462.1 & 395.5 & 287 & 5.08 \\
\hline 14 & 469.8 & 401.5 & 406 & 5.30 \\
\hline
\end{tabular}

Myristic acid

\begin{tabular}{llrc}
421.2 & 353.2 & 21 & 23.1 \\
421.2 & 356.5 & 11 & 12.1 \\
427.4 & 361.8 & 14 & 10.6 \\
429.0 & 363.6 & 27 & 18.5 \\
438.2 & 372.7 & 20 & 8.09 \\
445.8 & 380.2 & 53 & 14.2 \\
452.5 & 384.4 & 117 & 22.1 \\
463.8 & 394.9 & 146 & 15.7 \\
465.6 & 499.3 & 92 & 9.10 \\
473.5 & 408.1 & 117 & 8.03 \\
485.7 & 418.6 & 198 & 7.97 \\
\hline
\end{tabular}

supersaturation ratio. Alternatively, at large pressure ratios, e.g., greater than about 80 for heptanoic acid, the total density gradient inside the chamber reverses resulting in natural convection. The appropriate range of the pressure ratio must be determined for each working fluid.

In Figs. 1, 2, and 3 we plot the critical supersaturation data for heptanoic, decanoic, and myristic acids, respectively. The experimentally determined variation of the critical supersaturation with temperature is the envelope of the numbered curves while the continuous curves, labeled BDZ and KSR, represent the BeckerDöring-Zeldovitch and Katz-Saltsburg-Reiss predictions, respectively. The KSR plot is determined by setting the expression for the nucleation rate in Eq. (1) equal to unity and calculating the supersaturation $S$, as

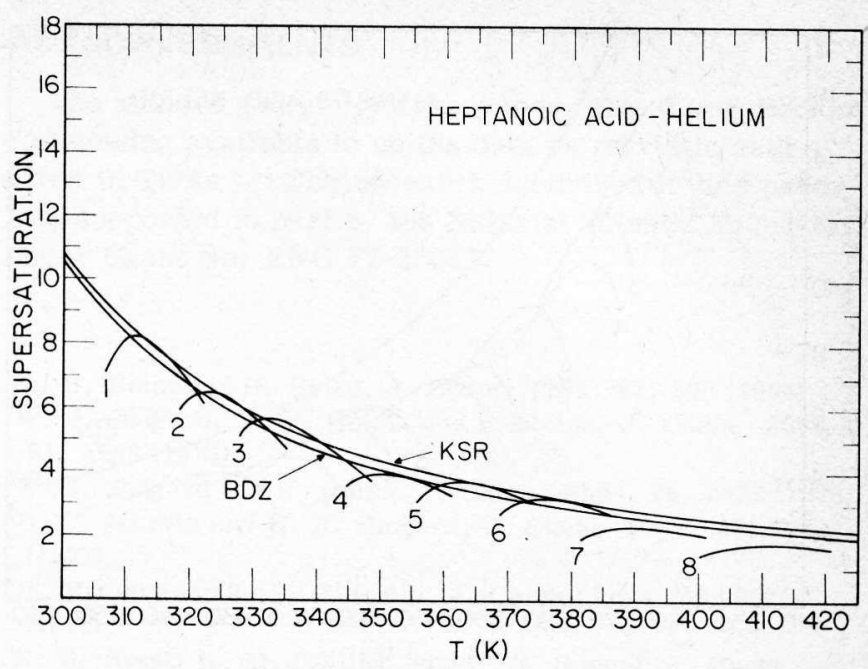

FIG. 1. The variation of the critical supersaturation of heptanoic acid vapor as a function of temperature. The curves labeled EDZ and KSR are the predictions of the Becker-Döring-Zeldovitch and Katz-Saltsburg-Reiss theories, respectively. The envelope of the numbered curves is the experimental result.

a function of temperature. The $\mathrm{BDZ}$ plot is calculated by setting the conventional homogeneous nucleation rate expression $^{11}$

$$
I=\frac{a}{d}\left(\frac{2 N_{0}^{3} \sigma M}{\pi}\right)^{1 / 2}\left(\frac{P_{e} S}{R T}\right)^{2} \exp \left[-\frac{16 \pi N_{0}}{3(\ln S)^{2}}\left(\frac{M}{d}\right)^{2}\left(\frac{\sigma}{R T}\right)^{3}\right]
$$

equal to unity and solving for the supersaturation as a function of temperature. In Eq. (4), $I$ is the homogeneous nucleation rate in drops $/ \mathrm{cm}^{3} / \mathrm{sec}$. The other symbols have been defined earlier.

For heptanoic acid, the degree of association is significantly less than for the formic, acetic, and propanoic acids discussed earlier. For instance, the dimer mole fraction at the critical supersaturation and $340 \mathrm{~K}$ for heptanoic acid is 0.06 as compared to the much larger

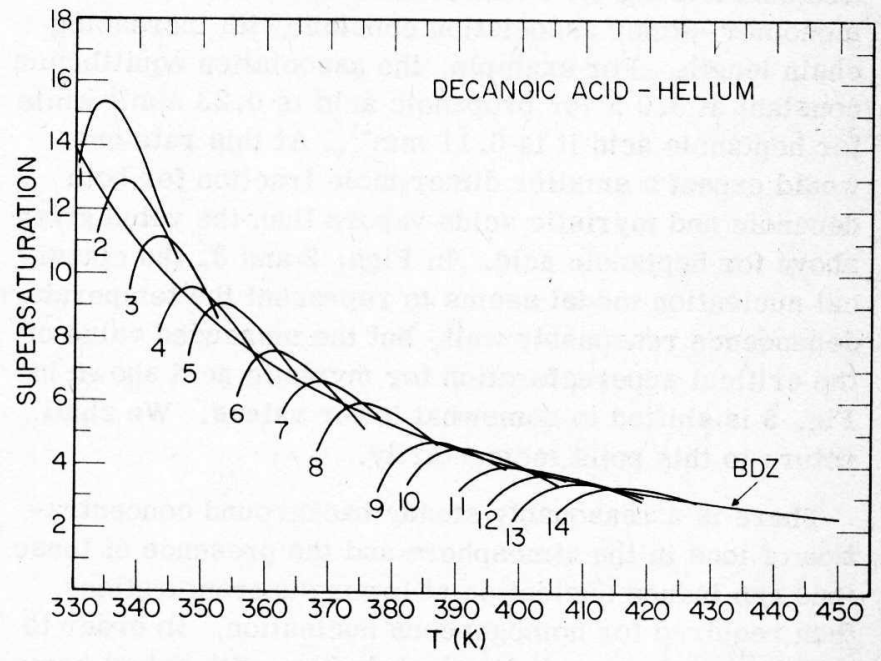

FIG. 2. The variation of the critical supersaturation of decanoic acid vapor as a function of temperature. The curve labeled BDZ is the prediction of the Becker-Döring-Zeldovitch theory. The envelope of the numbered curves is the experimental result. 


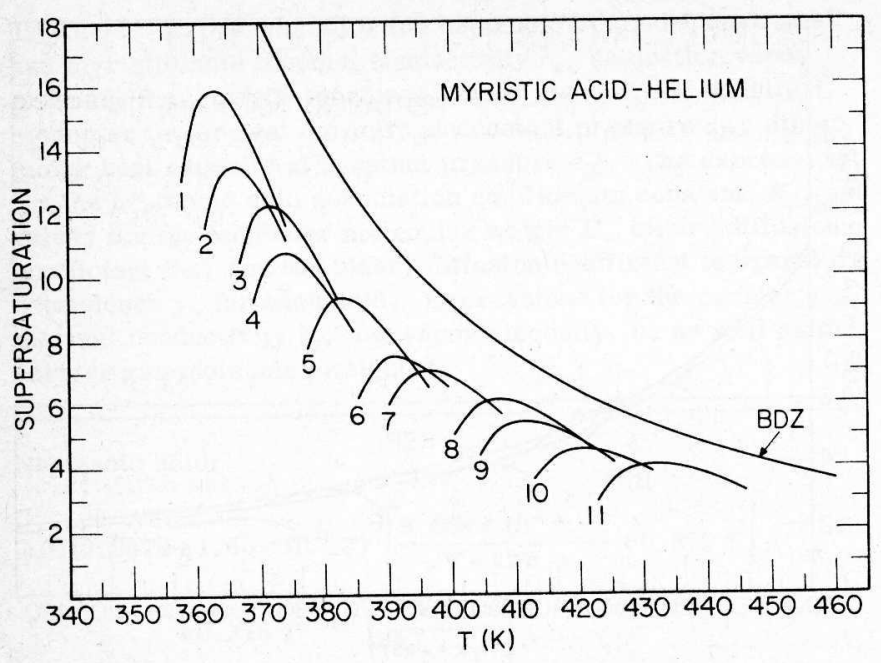

FIG. 3. The variation of the critical supersaturation of myristic acid vapor as a function of temperature. The curve labeled BDZ is the prediction of the Becker-Döring-Zeldovitch theory. The envelope of the numbered curves is the experimental result.

values given above for the lower molecular weight acids. This smaller degree of association is reflected in the fact that the measured critical supersaturation for heptanoic acid, as shown in Fig. 1, is very near the $\mathrm{BDZ}$ curve for a hypothetical vapor containing monomer only. For heptanoic acid the classical nucleation model appears to give a reasonable picture of both the temperature dependence and the measured values of the critical supersaturation. In Figs. 2 and 3 for decanoic and myristic acids, respectively, we have plotted only the BDZ curves because, as of yet, we have been unable to obtain satisfactory values for the monomer-dimer association equilibrium constants.

The numbered curves in Figs. 2 and 3 have been determined assuming a monomer-only vapor. While this is not strictly true, it seems a reasonable approximation considering the rate of decrease in the value of the monomer-dimer association constant with increasing chain length. For example, the association equilibrium constant at $310 \mathrm{~K}$ for propanoic acid is $0.23 \mathrm{~mm}^{-1}$ while for heptanoic acid it is $0.11 \mathrm{~mm}^{-1}$. At this rate one would expect a smaller dimer mole fraction for both decanoic and myristic acids vapors than the value given above for heptanoic acid. In Figs. 2 and 3 , the classical nucleation model seems to represent the temperature dependence reasonably well, but the measured value of the critical supersaturation for myristic acid shown in Fig. 3 is shifted to somewhat lower values. We shall return to this point momentarily.

There is a reasonably steady background concentration of ions in the atmosphere and the presence of these ions can induce nucleation at lower supersaturations than required for homogeneous nucleation. In order to avoid confusing ion-induced nucleation with actual homogeneous, we applied an electric field of $140 \mathrm{~V} / \mathrm{cm}$ across the cloud chamber plates to sweep out these naturally occurring ions. In fact, for the heptanoic, decanoic and myristic experiments, we were never able to observe any ion induced nucleation near the critical supersaturation. Interestingly, in all of our experiments involving carboxylic acids (see Refs 6 and 7), we have never been able to discern a change in the nucleation rate with or without an electric field applied across the chamber plates. Apparently, for the naturally occurring background concentration of charged particles, homogeneous nucleation always precedes ion-induced nucleation for these carboxylic acids.

A concern during any experiment involving carboxylic acids and metal cloud chamber plates is the possibility for chemical attack with adverse effect on chamber conditions. During all of our experiments with heptanoic, decanoic, and myristic acids, the aluminum cloud chamber plates never lost their original polish. We did observe, however, that after several days of experiments all of the acids were slightly discolored when removed from the chamber. The source of this discoloration is due apparently to some slight attack by the acids on the Viton gaskets used to separate the Pyrex ring from the aluminum plates. At the higher temperatures used in our measurements, e.g., 470-480 K, a small amount of effervescence could be observed along portions of the Viton gasket on the lower plate. However, we could find no relationship between our experimentally determined critical supersaturations and the histories of either the gaskets or the working fluid.

An increasingly serious problem in nucleation research involving the diff usion cloud chamber is the paucity of accurate thermodynamic and hydrodynamic data necessary to calculate the supersaturation profile inside the cloud chamber as well as the nucleation rate given by Eq. (1) or (4). As we move to increasingly complex chemical systems, we must rely more and more upon predictive techniques and empirical correlations to obtain this data. ${ }^{10}$ The carboxylic acids which were of interest in the three papers of this series are a good case in point. For instance, to obtain low temperature equilibrium vapor pressure data (below 1 Torr) for these higher molecular weight carboyxlic acids, we used both Cox chart and Dühring plot techniques ${ }^{12}$; it was necessary to estimate the vapor thermal conductivities of all these carboxylic acids ${ }^{13}$; group contribution techniques were used to estimate most of the monomer and dimer heat capacities ${ }^{13}$; vapor viscosities had to be estimated," as well as, the monomer-carrier gas binary diffusion coefficients. ${ }^{13}$ In addition, we have not yet been able to find experimental data or a reasonable predictive method for the association equilibrium constants for decanoic and myristic acids.

While in many cases these predictive methods are re sonably good, they introduce uncertainty into the results. ${ }^{10}$ The extent to which the solutions of Eqs. (2) and (3) depend upon the uncertainty of the values of the parameters listed in Table I has been discussed in detai elsewhere and need not be given here again. ${ }^{6,7,14}$ We shall comment, however, on one point which could have a significant effect upon the data shown in Figs. 1, 2, and 3. Because of the range of temperatures employed in our experiments, we were forced to extrapolate values of some of the properties beyond the range consid- 
ered valid for the existing experimental data. For instance, the available equilibrium vapor pressure data for heptanoic acid extended only to $351 \mathrm{~K}$ while the temperatures used in some of our experiments ranged below $300 \mathrm{~K}$. Similarly, for my ristic acid, the lower temperature limit of the available experimental data for the equilibrium vapor pressure was $390 \mathrm{~K}$ while the lower temperature range of our experiments was near $350 \mathrm{~K}$. In addition, the values of the extrapolated vapor pressures were small and very susceptible to the method of extrapolation. This is a serious problem since each of the numbered curves in Figs. 1, 2, and 3 are inversely proportional to the value of the equilibrium vapor pressure. For instance, if the estimated equilibrium vapor pressure is in error by $20 \%$ then the value of the experimental curve for the critical supersaturation will shift by a corresponding amount. Similarly, data for the bulk liquid surface tension was available for only a relatively narrow temperature range. This data had to be extrapolated using conventional techniques, beyond the temperature range of the data. This, too, is a serious problem since the bulk liquid surface tension enters as the cube in the exponential for the nucleation rate given in Eqs. (1) and (4). A $2 \%$ decrease in the surface tension, for example, lowers the BDZ curve for myristic acid by more than $12 \%$. This cannot explain the dramatic change in the critical supersaturation due to the monomer-dimer association reaction, but it could account for part of the apparent discrepancy in the myristic data in Fig. 3.

\section{ACKNOWLEDGMENTS}

The authors gratefully acknowledge Dr. Carol Becker for making available to us the data on myristic acid given in Table I. The research described in this paper was supported in part by the National Science Foundation under Grant No. ENG 77-25035.

${ }^{1}$ R. H. Heist and H. Reiss, J. Chem. Phys. 61, 573 (1974).

${ }^{2}$ W. J. Shugard, R. H. Heist, and H. Reiss, J. Chem. Phys. 61,5298 (1974).

${ }^{3}$ W. J. Shugard and H. Reiss, J. Chem. Phys. 65, 2827 (1976).

${ }^{4}$ D. C. Marvin and W. J. Shugard, J. Chem. Phys. 67, 5982 (1977).

${ }^{5}$ P. Mirabel and J. L. Clavelin, J. Chem. Phys. 68, 5020 (1978).

${ }^{6}$ R. H. Heist, K. M. Colling, and C. S. Dupuis, J. Chem. Phys. 65, 5147 (1976)

${ }^{7}$ Y. G. Russell and R. H. Heist, J. Chem. Phys. 69, 3723 (1978).

${ }^{8}$ J. L. Katz, H. Saltsburg, and H. Reiss, J. Colloid Interface Sci. 21, 560 (1966).

${ }^{9}$ R. H. Heist and H. Reiss, J. Chem. Phys. 59, 665 (1973).

${ }^{10} \mathrm{C}$. Becker, H. Reiss, and R. H. Heist, J. Chem. Phys. 68, 3585 (1978).

${ }^{11}$ See, for example, F. F. Abraham, Homogeneous Nucleation Theory (Academic, New York, 1974).

${ }^{12}$ See, for example, D. M. Himmelblau, Basic Principles and Calculations in Chemical Engineering (Prentice-Hall, New Jersey, 1974).

${ }^{13}$ For details, see Refs. 6 and 7 as well as Table $I$ in this paper.

${ }^{14}$ K. M. Colling, M. S. Thesis, University of Rochester, 1976. 